\title{
Development of the Optimal Design of Manual Garden Fork with the Ability to Quickly Clean the Working Surface
}

\author{
Ivan Voiku \\ Department of Business management \\ and innovation management \\ Pskov State University \\ Pskov, Russia \\ voiku-ivan@yandex.ru
}

\begin{abstract}
Manual garden tools help a lot with different types of work with soil: digging, removing roots and weeds, harvesting root crops and bulbs, and much more would be much more difficult if it were not for the forks. This tool has a wide range of features. There are many varieties of this tool differing in shapes and sizes: manure, harvesting, hay, flower, pointed, fork-shovel, telescopic, digging, ball-pointed. A common problem for all manual garden forks is clogging of the tines. This reduces the productivity of those who use them. Attempts are constantly being made to solve the problem of quick cleaning of the tines of manual garden forks. Many technical solutions to this problem are patented and are actively used in practice. The employee of the FSBEI of Higher Education "Pskov State University" has developed a manual self-cleaning fork. The design of the manual selfcleaning fork consists of a handle and a frame with many elongated spaced tines, which is fixed at one end of the handle, as well as a cleaning plate that is fixed to the movable handle, and a spring located on top of the handle inside the movable handle. The movable handle has lateral longitudinal grooves. The movable handle provides movement of the cleaning plate relative to the handle, and, consequently, compression and releasing of the spring. The overall dimensions of the proposed device are accepted according to the existing standards for such structures. The principle of operation of the proposed device: during the operation of the fork, the cleaning plate is pressed against the frame by spring force. Cleaning of the tines is made by a cleaning plate, which is moved along the tines. To do this, the user withdraws the movable handle, overcoming the resistance of the spring. By doing so, the frame passes through the longitudinal grooves and serves as one guide for the movable handle, and the handle serves as the second guide. The fork returns to its original state by releasing the spring after the user releases the movable handle. The main technical result of the
\end{abstract}

proposed device is an increase in the operational characteristics of the fork, reduction in the time and effort spent on cleaning of the tines.

Keywords - cleaning of tines, gardening, manual fork, manual labor.

\section{INTRODUCTION}

"Gardening” is defined as the activity of working in a garden, growing and taking care of plants, and keeping the garden attractive. [1] Gardening is a popular activity and a pastime in many countries and cultures. In the UK for example, it is estimated that $49 \%$ of the adult population takes part in gardening activities and that there are 24 million domestic (home) gardens in the UK; the country with a population of 66 million people. Similarly, in the USA, it is estimated that $78 \%$ of home-owners take part in gardening on a regular basis [1].

Despite the ongoing automation of modern production processes, manual labor continues to be necessary due to its flexibility and ease of deployment [3]. That is why, unlike large-scale agricultural activities, gardening involves manual labor, and, consequently, the use of manual agricultural tools.

The manual garden tools help a lot with different types of work with soil: digging, removing roots and weeds, harvesting root crops and bulbs, and much more would be much more difficult if it were not for the forks. This tool has a wide range of features.

There are many varieties of this tool, differing in shapes and sizes: manure, harvesting, hay, flower, pointed, fork-shovel, telescopic, digging, ball-pointed. 
The fertilization process is one of the stages that is very important for improving the quality and quantity of the crop. Manual forks are tools required for these agricultural operations. Their design is primarily developed for male workers taking their anthropometric body dimensions and strength parameters [4]. However, frequent use of manual forks even by men leads not only to a decrease in labor productivity, but also to health problems.

For example, in one study on tools for extracting components of the maize root system, an unscientific assessment of labor requirements estimates the productivity of using heavy garden forks at 28,6 roots/hour per person. While the use of tractor tools increases this figure to 92,4 roots/hour [5].

It's all about the common problem for all manual garden forks - clogging of the tines. This reduces the productivity of those who use them.

In recent years, many national governments and various stakeholders in the food value chain have purposefully introduced and used agricultural mechanization (AMT) technologies to stimulate agricultural production. With the advancement of technology, most Research and Development Institutes and Higher Educational Institutions (HEIs) involved in the agricultural sector of the country have been focusing on innovative technologies [2].

To facilitate tiresome work of cleaning of the tines of a manual fork, reduce corresponding health issues and increase labor productivity, the development of an ergonomic manual tool - manual self-cleaning fork - is in demand.

\section{MATERIALS AND METHODS}

There is a self-cleaning rake [Patent for invention US5713193 from 22.08.1996 "Self-cleaning rake" by Guinn Allen Y and Stapley Linda D (US)], consisting of a handle, a grip, a spring release button and two sets of tines [6]. The handle is made of fiberglass and consists of two telescoping pieces. One of the pieces is larger in diameter, and the other piece is slidably retained within it. The smaller handle piece is provided with a cleaning bar to which one set of tines are attached, and the cleaning bar further has a plurality of aligned apertures through which a second set of tines extend. The second set of tines are attached to the first handle piece, and reciprocable movement of the handle pieces relative to each other results in the cleaning bar and its attached tines moving in a manner which effects a removal of debris from both sets of tines.

The disadvantage of this device is that having two sets of tines leads to the removal of only large debris, and does not allow for full self-cleaning. In addition, a second set of tines significantly increase the weight of the device, reduce the effective area of the tines.

There is a device [Patent for invention US3884023(A) from 20.05.1975 “Self-cleaning rake” Robinson Melvin P (US)], which have tines with downwardly turned or depending front ends and is provided with a cleaning element slidably movable over the tines [7]. The cleaning element surrounds the tines and is pivotally connected to a bracket, which is in turn secured to a rod displaceable along the handle of the broom by means of a hand actautor. The cleaning element is designed to move along downwardly turned or depending front ends of the tines due to the pivotal connection to the bracket. The cleaning element also compresses the tines when it is moved downwardly over the tines.

The disadvantage of this device is that there is no return mechanism. In addition, cleaning of the tines involves compressing them, which in the case of heavy clogging can lead to deformation of the tines. The bracket located on top of the tines reduces the possibility of using this device in a confined space.

There is a self-cleaning rake [Patent for invention US4776158(A) from 11.10.1988 "Self-cleaning leaf rake" Baum Wendell E (US)], including an elongated handle and a frame with spaced tines, which is fixed at one end of the handle [8]. On the tines of the device there is a plate that is moved along the entire length of the tines by a push rod mounted on the handle. A spring clip holds the plate in the raised position and can be released by a guide element on the handle engaging the push rod.

The disadvantage of this device is the presence of a protruding push rod on the handle, as well as the lack of a return mechanism. To return the plate to its original state, the user must pause the process of using the device, must change the pose and position of the hands. The cleaning plate is moved by a push rod attached to the handle. The length of the handle, the lack of additional fasteners and items for stabilizing the position of the cleaning plate in case of severe clogging of the tines can lead to a device failure.

The closest to the claimed device is a self-cleaning rake [Patent for invention US2015257335 from 21.11.2014 "Self-cleaning rake” Riggs Christina and Riggs Donald (US)], which includes a hollow shaft and a frame with many elongated closely spaced tines, which is fixed at one end of the shaft [9]. Inside the shaft is a rod, at one end of which a cleaning plate is fixed, installed with the possibility of sliding along the tines. There is a grip on the outside of the shaft that is functionally connected to the rod. The handle allows you to move the cleaning plate along the tines. The rod is spring-loaded in such a way as to automatically return the cleaning plate to the frame.

The disadvantage of this device is low maintainability due to the location of the spring inside the hollow handle. Also, the use of a hollow shaft complicates the design and limits the list of materials for its manufacture. In addition, the location of the spring in the upper part of the handle increases material costs and reduces the usability due to the disruption of the user's familiar balance of the tool. In addition, this device cannot be created by upgrading the user's existing tools. In addition, to clean the tines the user must pause the process of using the device, change the pose and position of the hands. 


\section{RESULTS AND DISCUSSION}

The employee of the FSBEI of Higher Education "Pskov State University" has developed a manual selfcleaning fork [10].

The technical task solved by the claimed utility device is to increase maintainability, reduce material costs, increase usability, use standard materials, and ensure the possibility of upgrading existing tools.

The technical task is achieved by the fact that this manual self-cleaning fork consists of a handle and a frame with many elongated spaced tines, which is fixed at one end of the handle, a cleaning plate with many spaced holes in which the tines are located, a spring, a movable handle. The cleaning plate is fixed to the movable handle which has lateral longitudinal grooves, while the spring is located on top of the handle inside the movable handle.

The technical essence of the proposed device "Manual self-cleaning fork" is explained on Fig. 1 and Fig. 2.

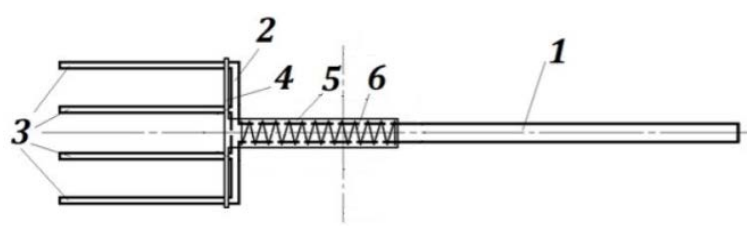

(a)

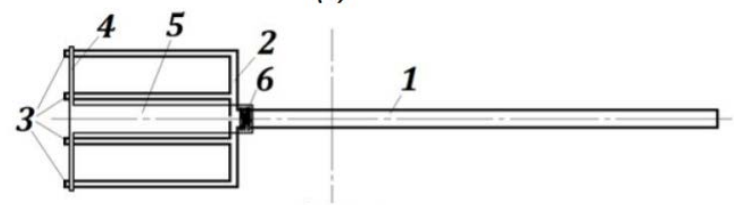

(b)

Fig. 1. Plan view of the manual self-cleaning fork (starting position (a) and working position (b)).

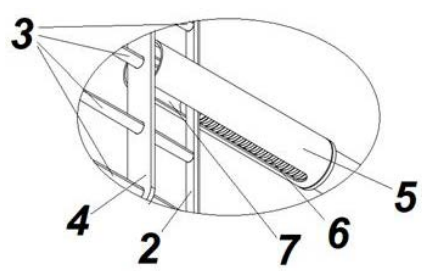

Fig. 2. Sketch of the movable handle of the self-cleaning fork as viewed from the teeth.

Essentially, the design of this manual self-cleaning fork consists of a handle 1 and a frame 2 with many elongated spaced tines 3 , which is fixed at one end of the handle 1 , as well as a cleaning plate 4 , which is fixed to the movable handle 5, and a spring 6 located on top of the handle 1 inside the movable handle 5 . The movable handle 5 has lateral longitudinal grooves 7.

The movable handle 5 provides the movement of the cleaning plate 4 relative to the handle 1 , and, consequently, the compression and releasing of the spring 6 . The overall dimensions of the proposed device are accepted according to the existing standards for such structures.
The principle of operation of the proposed device: during the operation of the fork, the cleaning plate 4 is pressed against the frame 2 by spring force 6 . Cleaning of the tines 3 is performed by the cleaning plate 4 , which is moved along the tines 3 . To do this, the user withdraws the movable handle 4, overcoming the resistance of the spring 6 . By doing so, the frame 2 passes through the longitudinal grooves 7 and serves as one guide for the movable handle 5 , and the handle 1 serves as the second guide. The fork returns to its original state by releasing the spring 6 after the user releases the movable handle 5 .

In the course of the experimental design work, the author of the device modeled two versions of mock-up samples (Fig. 3 and Fig. 4).

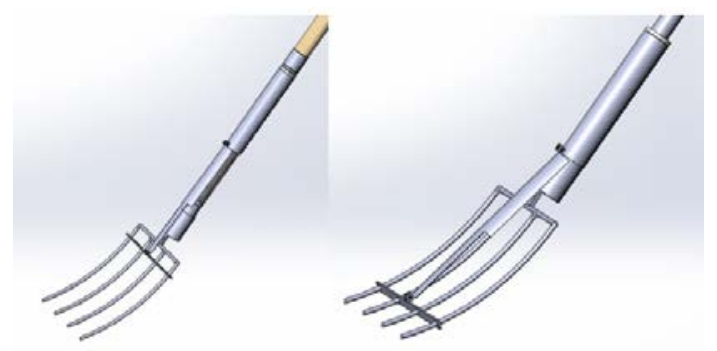

Fig. 3. Sketches of a mock-up sample of the manual self-cleaning fork (version 1).

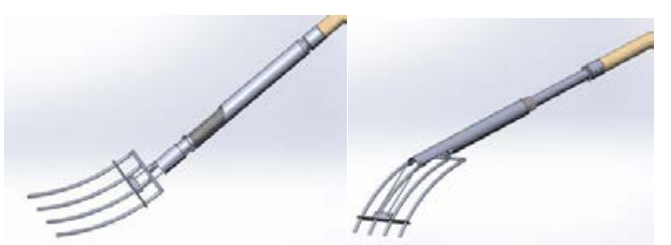

Fig. 4. Sketches of a mock-up sample of the manual self-cleaning fork (version 2).

Each of these versions differs in the direction of distribution of the user's force on the cleaning plate: above a plane of the tines (version 1) and below this plane (version 2). The results of the evaluation of the comparative effectiveness of the use of the design solutions will be presented based on the test results.

In general, the technical result of the claimed utility device is an increase in the operational characteristics of the self-cleaning fork, reduction in the time and effort spent on cleaning of the tines.

\section{CONCLUSIONS}

Gardening is a popular activity and a pastime in many countries and cultures.

Despite the ongoing automation of modern production processes, manual labor continues to be necessary due to its flexibility and ease of deployment.

Manual garden tools help a lot with different types of work with soil: digging, removing roots and weeds, harvesting root crops and bulbs, and much more would be much more difficult if it were not for the forks. 
A common problem for all manual garden forks is clogging of the tines. This leads to a decrease in the productivity of those who use them.

In the course of the research work, patented scientific and technical developments aimed at solving the problem of quick cleaning of the tines of forks, rakes and other similar devices were identified. They all have their advantages and disadvantages.

The employee of the FSBEI of Higher Education "Pskov State University" has developed a manual selfcleaning fork.

Essentially, the design of the manual self-cleaning fork consists of the handle having at one end the frame with many elongated spaced tines, as well as the cleaning plate, which is fixed to the movable handle, and the spring located on top of the handle inside the movable handle. The movable handle has lateral longitudinal grooves. The movable handle provides the movement of the cleaning plate relative to the handle, and, consequently, compression and releasing of the spring.

The principle of operation of the proposed device is simple: cleaning of the tines is performed by the cleaning plate which is moved along the tines. To do this, the user withdraws the movable handle, overcoming the resistance of the spring. The fork returns to its original state by releasing the spring after the user releases the movable handle.

In the course of the experimental design work, the author of the device modeled several versions of the mockup samples. In general, the technical result of the claimed device is an increase in the operational characteristics of the self-cleaning fork, reduction in the time and effort spent on cleaning the tines. The manual self-cleaning fork allows a gardener to clean the fork's tines from clogging with a single movement of the hand.

\section{REFERENCES}

[1] L. Chalmin-Pui, A. Griffiths, J. Roe, T. Heaton, and R. Cameron, "Why garden? - Attitudes and the perceived health benefits of home gardening," Cities, vol. 112, p. 103+, May 2021. [Online]. Available: ScienceDirect, https://www.sciencedirect.com/ [Accessed February 2021] https://doi.org/10.1016/j.cities.2021.103118

[2] R. M. Amongo, E. Quilloy, M. A. Ranches, M. V. Larona, and M. Madlangbayan, "Development of an electric hand tractor (eTractor) for agricultural operations," IOP Conference Series: Earth and Environmental Science, vol. 542, p. 012+, 2020. [Abstract]. Available: https://doi.org/10.1088/1755-1315/542/1/012027

[3] C. Löffler, C. Nickel, C. Sobel, D. Dzibela, J. Braat, B. Gruhler, P. Woller, N. Witt, and C. Mutschler, Automated Quality Assurance for Hand-Held Tools via Embedded Classification and AutoML, 2021. [Abstract]. Available: Springer link, https://link.springer.com/, https://doi.org/10.1007/978-3-030$\underline{67670-4 \quad 33}$

[4] S. Chitraputhira Pillai and P. Surya, Drudgery Reduction Through Use of Hand Held Fertilizer Applicator, 2020.

[5] T. Praiswater, B. Hibbard, B. Barry, L. Darrah, and V. Smith, "An Implement for Dislodging Maize Roots from the Soil for Corn Rootworm (Coleoptera: Chrysomelidae) Damage Evaluations," Journal of the Kansas Entomological Society, vol. 70, pp. 335-338, 1997.

[6] A. Y. Guinn and L. D. Stapley, "Self-cleaning rake," U. S. Patent 5,713,193, August 22, 1996

[7] M. P. Robinson, "Self-cleaning rake," U. S. Patent 3,884,023(A), May 20, 1975.

[8] W. E. Baum, "Self-cleaning leaf rake," U. S. Patent 4,776,158(A), October 11, 1988.

[9] C. Riggs and D. Riggs, "Self-cleaning rake," U. S. Patent 2,015,257,335, November 21, 2014.

[10] I. Voiku, "Manual self-cleaning fork," R. U. Patent 199,181 U1, February 10, 2020. 